\title{
HPV vaccine in the treatment of usual type vulval and vaginal intraepithelial neoplasia: a systematic review
}

\author{
Stacey Bryan ${ }^{1 *}$ (D), Cynthia Barbara², Jane Thomas² and Adeola Olaitan²
}

\begin{abstract}
Background: HPV DNA is found in almost $80 \%$ of VINNalN. Current management is inadequate, with high recurrence rates. Our objective was to review the literature regarding the role of HPV vaccine in secondary prevention and treatment of VIN/NaIN.

Methods: Database searches included Ovid Medline, Embase, Web of Science, The Cochrane Library and Clinicaltrials.gov. Search terms included HPV vaccine AND therapeutic vaccine* AND VIN OR VAIN, published in English with no defined date limit. Searches were carried out with a UCL librarian in March 2018. We included any type of study design using any form of HPV vaccine in the treatment of women with a histologically confirmed diagnosis of VIN/NaIN. We excluded studies of other lower genital tract disease, vulval/vaginal carcinoma and prophylactic use of vaccines. The outcome measures were lesion response to vaccination, symptom improvement, immune response and HPV clearance.

Results: We identified 93 articles, 7 studies met our inclusion criteria; these were uncontrolled case series. There were no RCTs or systematic reviews identified. Reduction in lesion size was reported by all 7 studies, symptom relief by 5 , HPV clearance by 6 , histological regression by 5 , and immune response by 6 .

Conclusions: This review finds the evidence relating to the use of HPV vaccine in the treatment of women with VIN/ ValN is of very low quality and insufficient to guide practice. Further longitudinal studies are needed to assess its use in prevention of progression to cancer.
\end{abstract}

Keywords: HPV vaccine, VIN, VAIN, Therapeutic vaccine, Vulval disorders, Vulvar intraepithelial neoplasia

\section{Background}

Vulval/Vaginal Intraepithelial Neoplasia (VIN/VaIN) can precede the development of invasive cancer by a variable period. Usual-type VIN (uVIN) is commonly associated with carcinogenic subtypes of human papillomavirus (most commonly HPV 16) [1]. The HPV attribution for different anogenital lesions varies between studies, however, is found in approximately $76-87 \%$ of VIN, $30-40 \%$ of vulvar cancer [1], 96\% of VaIN and $63-74 \%$ of vaginal cancer $[2,3]$.

The incidence of VIN is thought to be around 1-2/ 100,000 [4]. Although spontaneous regression has been reported (approx. 1.2\%), VIN is considered a premalignant condition (as is VaIN), with a suggested progression

\footnotetext{
* Correspondence: sjbryan@doctors.org.uk

${ }^{1}$ Research Fellow, Institute for Women's Health, Gynaecology Cancer

Research Centre, 1st Floor Maple House, 149 Tottenham Court Road, London WIT 7NF, UK

Full list of author information is available at the end of the article
}

rate to cancer of 3-16\% [5]. Although primarily seen in older women (over 70 years old), $15 \%$ are diagnosed in women under the age of 50 . Although presentation of VIN may be asymptomatic, symptoms often include pruritis, burning, and pain which can be distressing [6] In addition, lesions may be multifocal affecting large areas of the vulva, pigmented or white or present as areas of erythema. Lesions may also be flat, raised or ulcerated [5].

Vaginal Intraepithelial neoplasia is an uncommon (incidence around $0.1 / 100,000$ women in the US) usually asymptomatic disease [7], often associated with other intraepithelial neoplasias. Progression to invasive vaginal cancer has been reported as approximately $2 \%$ [8].

The current treatments for VaIN are either surgical (excision or $\mathrm{CO}_{2}$ ablation) or medical (Imiquimod, 5FU or vaginal oestrogens) [9]. Most treatment regimens for

(c) The Author(s). 2019 Open Access This article is distributed under the terms of the Creative Commons Attribution 4.0 International License (http://creativecommons.org/licenses/by/4.0/), which permits unrestricted use, distribution, and reproduction in any medium, provided you give appropriate credit to the original author(s) and the source, provide a link to the Creative Commons license, and indicate if changes were made. The Creative Commons Public Domain Dedication waiver (http://creativecommons.org/publicdomain/zero/1.0/) applies to the data made available in this article, unless otherwise stated. 
VIN also consist of ablation or excision, medical therapy with Imiquimod has been shown to be effective [10] and is commonly used by dermatologists and genitourinary medicine (GUM) specialists. Its use can be limited by intolerable side effects such as erythema and vulval pain. Recurrence rates after treatment, regardless of modality are as high as $30-50 \%$, implying that current treatment options are inadequate and not effective in the long term [11]. A review of practice at our unit showed poor response rate to all treatment modalities with rate of progression to invasive disease at $16 \%$. Almost $15 \%$ of patients attend for 10 years or more, undergoing multiple biopsies and/or surgical treatments, in addition to being on long term Imiquimod treatment. These women participate in multiple hospital visits which is a burden to them and increases healthcare services costs. These findings from our own practice prompted this systematic review.

Women with natural antibodies to HPV have less frequent recurrence of VIN than those who are antibody negative $(22.9 \%$ vs $52 \%)$ [12]. Three vaccines are available for the primary prevention of HPV infection. A bivalent vaccine (Cervarix ${ }^{\oplus}$ [GSK]) targets high risk HPV types 16 and 18 , whilst a quadrivalent vaccine (Gardasil ${ }^{\circ}$ ) targets the same HR HPV types (16 and 18) and two HPV types that commonly cause genital warts $(6,11)$. A newer recombinant 9-valent vaccine prepared from the purified virus-like particles (VLP) of the major capsid (L1) protein of the 9 HPV subtypes - 6, 11, 16, 18, 31, $33,45,52,58$, is now available (Gardasil 9 [MSD UK]). These are widely used for the prevention of premalignant genital lesions, and premalignant anal lesions, in females and males. These appear to be most effective at preventing CIN in women who are known to be HRHPV negative, but there is moderate to high quality evidence that they reduce high grade CIN for women whose HPV status is not known [13-15]. This may be because prophylactic vaccination has been shown to augment the response to HPV in women who were previously seropositive [16]. This is consistent with studies that indicate that vaccination may have a therapeutic effect in other HPV-associated conditions such as recurrent respiratory papillomatosis (RRP), a benign neoplasm of the larynx among children, caused by HPV 6 and 11 [17].

Prophylactic vaccines act by inducing neutralising antibodies to the viral L1 capsid proteins. It is thought that treatment of already established HPV infection requires activation of T-cells capable of killing virus infected cells, rather than producing antibodies against the virus itself, so therapeutic vaccines (in development), target viral genome E6 and E7 oncoproteins (necessary for initiation and maintenance of transformation to cancer) [18].

Different types of therapeutic vaccines have been tested in other HPV driven diseases. These include;
- Protein-based vaccines, which can be long or short peptide chains, and comprise TA-CIN (Tissue Antigen Cervical Intraepithelial Neoplasia) a fusion protein of HPV 16 L2, E6 and E7 [18, 19];

- DNA based vaccines that induce cytotoxic lymphocytes, T helper cells, and increase B cell immunity;

- A vaccine containing a mixture of two plasmids encoding HPV 16/18 E6 and E7 antigens;

- TA-HPV (therapeutic antigen HPV) a vaccinia vector-based vaccine expressing modified forms of HPV 16 and 18 E6 and E7 proteins and

- Cell-based vaccines such as dendritic cells and tumour infiltrating T-cells [18].

The currently licensed HPV vaccines have an established role in the primary prevention of HPV infection generally in younger populations. The role of the HPV vaccine in secondary prevention and treatment has not yet been fully established. Women with VIN may have symptoms which are recurrent and difficult to treat. Clinicians who treat these patients (dermatologists, GUM specialists and gynaecologists) look for ways to improve the effectiveness of current treatment.

We performed a review of the literature regarding the role of any HPV vaccination for secondary prevention, in the treatment of women with HPV-related VIN and VaIN.

\section{Methods}

We conducted a systematic search of the literature with the strategy to review best available evidence. We searched for systematic reviews, and randomized controlled trials. If these were not available, we would include controlled non-random studies, and uncontrolled studies. We looked into the use of any form of HPV vaccine in the treatment of women with a histologically confirmed diagnosis of HPV related VIN and/or VaIN, versus control/standard treatment. These vaccines included the commercially available licensed vaccines prepared from the VLPs of the L1 protein of common HPV types, or the experimental vaccines against the HPV oncoproteins E6/E7. Our search also included vaccines as adjuvant to usual care. We excluded studies of other lower genital tract disease (pre-invasive or invasive), vulval/vaginal carcinoma and those studies concerning prophylactic use of vaccines.

We excluded studies whose participants were pregnant, immunocompromised, or had a history of allergy to vaccine products. In the included studies, patients with VIN or VaIN were given any form of HPV vaccination.

The outcome measures we wished to evaluate were lesion response to vaccination, symptom improvement, immune response, and HPV clearance. A Core Outcome Set protocol was not used as this is not currently available but is in development for vulva. 
Database searches included Ovid Medline, Embase, Web of Science, The Cochrane Library and Clinicaltrials.gov. Search terms included HPV vaccine OR Human Papilloma Virus vaccine OR Papilloma virus vaccines OR HPV Vac-

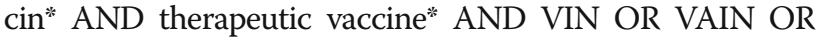
Vulval intraepithelial neoplasia OR Vaginal intraepithelial neoplasia, published in English with no defined date limit. Database searches were carried out with the aid of a UCL librarian in March 2018. Additional papers were identified via the reference lists of included studies (Additional file 1).

\section{Results}

Our search strategy is detailed in the Prisma flow diagram in Additional file 2: Figure S1. Two independent reviewers identified 93 articles after excluding studies with titles unrelated to the subject matter. A further 86 articles were excluded at this stage: 55 were review articles i.e. not primary research or not addressing the review question. Seventeen studies were excluded as they reported on experimental HPV vaccine development - identifying the right dose for effect or analyzing the types of immune response to HPV vaccination. Four studies were concerned with HPV vaccine as prevention not as treatment and three studies were concerned with effect of experimental HPV vaccine in non-human subjects. In a search of clinicaltrials.org we found 4 potential studies - two were active trials but not yet recruiting, with results expected in late 2018 - one involved CIN only and the other, a study from China which had completed, but no results had been reported at the time of writing. Two studies were concerned with other forms of anogenital pre-cancer and 1 study was concerned with trial of Imiquimod only.

There were no RCTs, systematic reviews or controlled studies identified. Seven studies that included 129 women, met our inclusion criteria, the included studies were all uncontrolled case series published between 2000 and 2017 (Table 1).

\section{Study participants}

Of the 129 participants 126 had VIN and 3 had VAIN [19-25]. Duration of disease (VIN/VAIN) ranged from 2 months to 17 years, with approximately $60 \%$ of patients having had previous treatment(s). In two of the studies the women had a mean age of 29 whilst the other studies' participants were over the age of 40 years. The studies showed heterogeneity in the number of patients included (12 to 29) and disease duration prior to vaccination ( 2 months to 17 years), whilst the follow up was equally short (1-12 months) in all.

\section{Intervention}

None of the studies used the commercially available vaccines. All of the studies used experimental vaccines of either TA-HPV (therapeutic antigen HPV) a vaccinia-based vector vaccine, or TA-CIN (Tissue Antigen Cervical Intraepithelial Neoplasia), a fusion protein vaccine comprising HPV16 viral proteins L2, E6 and E7 - the latter 2 responsible for inactivation of the tumour suppressor proteins $\mathrm{p} 53$ and $\mathrm{pRb}$ respectively, leading to hyper-proliferation of host cells and overexpression of p16 and p14 [5], which can lead to cancer.

\section{Outcomes}

Change in lesion size pre and post HPV vaccination was an outcome reported by all 7 studies. Symptom relief was reported by 5 studies, HPV clearance by 6 studies, histological regression by 5 studies, and immune response by 6 of the 7 studies. Follow-up was short - most commonly from 1 month to 1 year.

\section{Lesion response to experimental HPV vaccination}

Regression of the lesion was assessed by measuring lesion size before and after vaccination, commonly in two dimensions and taking the average of both sizes. Most of the studies categorized these into partial response (if more than a $50 \%$ reduction in size), no response (if less than $50 \%$ reduction) or complete response if no lesion was visible following vaccination. In these studies, lesion regression varied from no response up to an overall partial and complete response of $83 \%$.

\section{Symptom relief}

Clinical improvement in symptoms was measured by either direct questioning at each visit or by asking participants to keep a symptom diary. Although no formal validated tool was used, commonly, symptoms were subjectively graded into none/mild/moderate/severe, depending on how it affected patient's daily lives. The effects on symptom relief varied from no overall change in symptoms to $68.4 \%$ of women becoming symptom free after the follow up period.

\section{Immune response to experimental HPV vaccination}

Immune response to vaccination ranged from 30 to $94 \%$. Various different markers were used to determine immune responses ( $\mathrm{T}$-cell responses vs lymphoproliferative vs cytokine). The types of experimental vaccines, schedules and in some cases delivery method, also differed and this may contribute to the heterogeneity seen between study findings.

\section{HPV clearance}

HPV clearance is defined as testing HPV negative post vaccination having previously tested positive. The rate of HPV clearance varied between 8 and $74 \%$. Histological regression is defined as the index VIN/VaIN lesion being downgraded from a high grade to a low-grade lesion. The rate of this occurring varied from 0 up to $63 \%$. HPV 


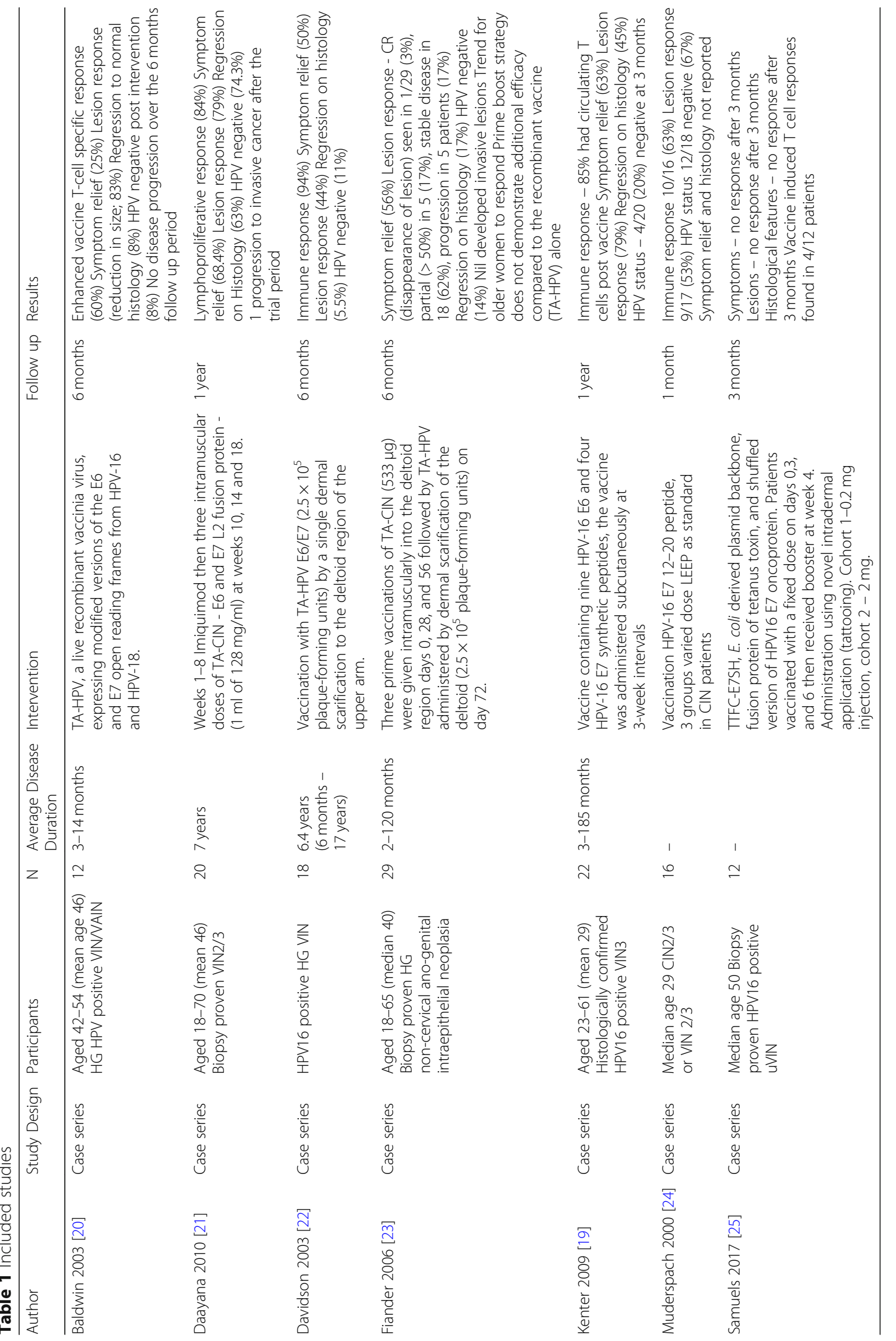


status and histological regression did not seem to correlate with regression of the lesion nor the patient's symptoms.

There was a wide variation in results in relation to the above outcomes, which may reflect the differences in patients and the types of vaccines and schedules used.

\section{Discussion}

This study is the first systematic review of the use of any HPV vaccination in the treatment of HPV related vulval and vaginal disorders. This review has highlighted that no high-quality studies using commercially available vaccines have addressed this research question - available vaccines are designed for prevention. Studies that have been carried out are small, case series without control groups, rather than randomized controlled trials. They have used experimental therapeutic vaccines rather than the available vaccines. The study designs are of low methodological quality and a high risk of bias. Systematic reviews of randomized control trials indicate that available HPV vaccines are safe and effective for prevention. Effectiveness seems to decrease with age and exposure to HPV. The role of these vaccines as adjuvant to treatment has not been studied. The efficacy and safety of the experimental vaccines is yet to be established and it is likely to be a number of years before therapeutic vaccines become available for clinical use. Overall the evidence to answer the clinical questions of the role of HPV vaccination in the treatment of VIN/VAIN is of very low-quality.

\section{Clinical responses to experimental HPV vaccine}

When comparing other systematic reviews and studies evaluating treatment outcomes for vulval disease, reporting centers primarily on response to treatment, with response being described as resolution, persistence or progression. This could be assessed clinically as a reduction in size of the lesion, symptoms response as reported by the patients, histological resolution (this assessment is invasive because it requires repeat biopsies), HPV clearance or persistence.

A number of patients experienced relief of their symptoms post vaccination. A large number also experienced a reduction in the size of their lesions - it is unclear whether this correlates with symptom relief. Current treatments for VIN may be effective in the short term but with significant side effects and a high risk of recurrence.

Of the studies which we identified, one reported no response in any of the initial outcome measures after 3 months [25]. In this study the novel technique of intradermal tattooing was used. This technique has been evaluated in animal models and the efficacy compared to traditional intramuscular vaccination has not been established in humans. The method of vaccination may have contributed to these negative results.

\section{Strengths and limitations}

Our review examines the role of HPV vaccination as an adjuvant to treatment for women who have HPV related VIN/VaIN. Whilst Miltz et al have examined the role of vaccination in anogenital pre-cancer [26], our review differs in that it focuses on vulval and vaginal diseases rather than cervical/CIN. We were interested in exploring interventions that could be used alongside existing treatments for VaIN and VIN to improve effectiveness and/ or reduce recurrence and need for further treatments. We used a systematic approach to search library databases. None of the studies directly compared a control group with HPV vaccination, all studies found were uncontrolled case series, with short follow up for what is a chronic, long term disease. We were therefore limited by the level of evidence with which to answer the question.

\section{Conclusion}

Women with VIN/VaIN are living with chronic conditions, requiring prolonged and often repeated treatments which can have intolerable side effects, and yet high relapse rates. More effective treatments are needed that will reduce symptoms, reduce progression and reduce the rate of relapse. Having been introduced almost a decade ago as part of the childhood vaccination program, we should begin to see a reduction in the incidence of VIN/VAIN and other anogenital pre-cancers. In the meantime, for patients who have not been vaccinated, newer treatments with fewer side effects and morbidity are warranted. The evidence for the use of HPV vaccination in VIN/VaIN is of low quality and insufficient to guide practice. All of the vaccines used in these non-randomized studies were experimental and we did not find any research evaluating the use of commercially available vaccine as an adjuvant to usual treatment of VAIN/VIN. We aim to deliver such a study following on from this review. More studies are needed to investigate clinically important patient outcomes (symptoms, non-progression and recurrence rates) with the use of HPV vaccine in a therapeutic setting. It would be prudent to also investigate its use in secondary prevention of cancer, which would need to be assessed through further longitudinal studies.

\section{Additional files}

Additional file 1: Appendix S1 - searches. (PDF $516 \mathrm{~kb}$ )

Additional file 2: Figure S1 - Prisma Flow Diagram. (DOCX 108 kb)

\section{Acknowledgements}

Thanks to Julia Garthwaite, Deputy Librarian, for assisting in the literature searches for this review.

Funding

Not applicable 


\section{Availability of data and materials}

All data generated or analysed during this study are included in this published article and its supplementary information files. This review has been prospectively registered in PROSPERO (CRD42018093463).

\section{Authors' contributions}

SB performed the literature search with the librarian, selected the studies and wrote the paper. CB reviewed the search results, checked for inclusion and exclusion criteria and reviewed the studies, acting as a second reviewer. JT provided support with regards to study designs, selections, inclusions/ exclusion criteria, structuring the review recording and presenting the outcomes, and also proof reading and editing the manuscript. AO provided the review idea and topic, clinical details, audit data and also proofread and edited the manuscript. All authors read and approved the final manuscript.

\section{Ethics approval and consent to participate}

Not applicable

\section{Consent for publication}

Not applicable

\section{Competing interests}

The authors declare they have no competing interests.

\section{Publisher's Note}

Springer Nature remains neutral with regard to jurisdictional claims in published maps and institutional affiliations.

\section{Author details}

${ }^{1}$ Research Fellow, Institute for Women's Health, Gynaecology Cancer Research Centre, 1st Floor Maple House, 149 Tottenham Court Road, London W1T 7NF, UK. ${ }^{2}$ University College London Hospitals, 2nd Floor North, 250 Euston Road, London NW1 2PG, UK.

Received: 14 August 2018 Accepted: 28 December 2018

Published online: 07 January 2019

\section{References}

1. Faber MT, Sand FL, Albieri V, Norrild B, Kjaer SK, Verdoodt F. Prevalence and type distribution of human papillomavirus in squamous cell carcinoma and intraepithelial neoplasia of the vulva. Int J Cancer. 2017;141(6):1161-9.

2. Cancer Research UK, "Cancer research UK," [Online]. Available: https://www. cancerresearchuk.org/health-professional/cancer-statistics/statistics-bycancer-type/vaginal-cancer. Accessed 11 May 2018.

3. Serrano B, de Sanjose S, Tous S, Quiros B, Munoz N, Bosch X, Alemany L. Human Papillomavirus genotype attribution for HPVs 6, 11, 16, 18, 31, 33, 45, 52 and 58 in female anogenital lesions. Eur J Cancer. 2015;51(13):1732-41.

4. National Cancer Institute, Surveillance Research Program, Cancer Statistics, "Surveillance, Epidemiology, and End Results (SEER) program," 2006. [Online]. Available: www.seer.cancer.gov. Accessed 11 May 2018.

5. Del Pino M, Rodriguez-Carunchio L, Ordi J. Pathways of vulval intraepithelial neoplasia and squamous cell carcinoma. Histopathology. 2013;62:161-75.

6. Reyes C, Cooper K. An update on vulvar intraepithelial neoplasia: terminology and a practical approach to diagnosis. J Clin Pathol. 2014;67:290-4.

7. Cramer DW, Cutler SJ. Incidence and histopathology of malignancies of the female genital organs in the United States. Am J Obstet Gynecol. 1974;118: 443-60

8. Dodge J, Eltabbakh GH, Mount SL, Walker RP, Morgan A. Clinical features and risk recurrence among patients with Vaginal Intraepithelial Neoplasia. Gynecol Oncol. 2001:83:363-9.

9. Tranoulis A, Laios A, Mitsopoulos V, Lutchman-Singh K, Thomakos N. Efficacy of $5 \%$ Imiquimod for the treatment of Vaginal Intraepithelial Neoplasia - a systematic review of the literature and meta-analysis. Eur J Obstet Gynecol Reprod Biol. 2017;218:129-36.

10. Pepas L, Kaushik S, Nordin A, Bryant A, Lawrie TA. Medical interventions for high-grade vulval intraepithelial neoplasia, vol. 8: Cochrane Database Syst Rev; 2015. p. CD007924.

11. Lawrie TA, Nordin A, Chakrabarti M, Bryant A, Kaushik S, Pepas L. Medical and surgical interventions for the treatment of usual-type vulval intraepithelial neoplasia. Cochrane Database Syst Rev. 2016;(1). Art. No.: CD011837. https://doi.org/10.1002/14651858.CD011837.pub2.
12. Madeleine MM, Johnson LG, Doody DR, Tipton ER, Carter JJ, Galloway DA. Natural antibodies to human papillomavirus 16 and recurrence of vulvar high grade intraepithelial neoplasia (VIN3). J Low Genit Tract Dis. 2016;20(3): 257-60.

13. Costa APF, Cobucci RNO, da Silva JM, da Costa Lima PH, Giraldo PC, Gonçalves AK. Safety of human papillomavirus 9-valent vaccine: A metaanalysis of randomized trials. J Immunol Res. 2017;2017:1-6.

14. Vesikari T, Brodszki N, van Damme P, Diez-Domingo J, Icardi G, Petersen L, Tran C, Thomas S, Luxembourg A, Baudin M. A randomized, double blind, phase 3 study of the immunogenicity and safety of a 9-valent human papillomavirus L1 virus-like particle vaccine (V503) versus Gardasil in 9-15year-old girls. Pediatr Infect Dis J. 2015;34(9):992-8.

15. Arbyn M, Simoens C, Martin-Hirsch PP. Prohylactic vaccination against human papillomaviruses to prevent cervical cancer and its precursors (review). Cochrane Database Syst Rev. 2018:5:CD009069.

16. Olsson S-E, Villa LL, Costa RL, Petta CA, Andrade RP, Malm C, Iversen O-E, Hoye J, Steinwall M, Riis-Johannessen G, Andersson-Ellstrom A, Elfgren K, von Krogh $\mathrm{G}$, Lehtin $\mathrm{M}$. Induction of immune memory following administration of a prophylactic quadrivalent human papillomavirus (HPV)types 6/11/16/18 L1 virus-like (VLP) vaccine. Vaccine. 2007;25:4931-9.

17. Chirila M, Bolboaca SD. Clinical efficiency of quadravalent HPV (types 6/11/ 16/18) vaccine in patients with recurrent respiratory papillomatosis. Eur Arch Otorhinolaryngol. 2014;271:1135-42.

18. Vici P, Pizzuti L, Mariani L, Zampa G, Santini D, Di Lauro L, Gamucci T, Natoli C, Marchetti P, Barba M, Maugeri-Sacca M, Sergi D, Tomao F, Vizza E, Di Fillippo S, Paolini F, Curzio G, Corrado G, Michelotti A, Sanguineti G, Giordano A. Targeting immune response with therapeutic vaccines in premalignant lesions and cervical cancer: hope or reality from clinical studies. Expert Review vaccines. 2016;15(10):1327-36.

19. Kenter GG, Welters MJ, Valentijn RP, Lowik MJ, Berends-van der Meer DM, Vloon AP, Essahsah F, Fathers LM, Offringa R, Drijfhout JW, Wafelman AR, Oostendorp J, Fleuren G. Vaccination against HPV-16 oncoproteins for vulvar intraepithelial neoplasia. N Engl J Med. 2009;361:1838-47.

20. Baldwin PJ, Van der Burg SH, Boswell CM, Offringa R, Hickling JK, Dobson J, Roberts JS, Latimer JA, Moseley RP, Coleman N, Stanley MA, Sterling JC. Vaccinia-expressed human papillomavirus 16 and 18 E6 and E7 as a therapeutic vaccination for vulval and vaginal intraepithelial neoplasia. Clin Cancer Res. 2003;9(14):5205-13.

21. Daayana S, Elkford E, Winters U, Pawlita M, Roden R, Stern PL, Kitchenr HC. Phase 2 trial of Imiquimod and HPV therapeutic vaccination in patients with vulval intraepithelial neoplasia. Br J Cancer. 2010;102(7):1129-36.

22. Davidson EJ, Boswell CM, Sehr P, Pawlita M, Tomlinson AE, McVey RJ, Dobson J, Roberts JS, Hickling J, Kitchener HC, Stern PL. Immunological and clinical responses in women with vulval intraepithelial neoplasia vaccinated with a vaccinia virus encoding HPV 16/18 oncoproteins. Cancer Res. 2003; 63(18):6032-41.

23. Fiander AN, Tristram AJ, Davidson EJ, Tomlinson AE, Man S, Baldwin PJ, Sterling JC, Kitchener HC. Prime-boost vaccination strategy in women with highgrade, noncervical anogenital intraepithelial neoplasia: Clinical results from a multicenter phase II trial. Int J Gynaecological Cancer. 2006;16:1075-81.

24. Muderspach L, Wilczynski S, Roman L, Bade L, Felix J, Small L, Kast WM, Fascio G, Marty V, Weber J. A phase 1 trial of human papillomavirus (HPV) peptide vaccine for women with high grade cervical and vulvar intraepithelial neoplasia who are HPV 16 positive. Clin Cancer Res. 2000;6(9):3406-16.

25. Samuels S, Marijne Heeren A, HJMAA Z, MJP W, van den Berg JH, Philips D, Kvistborg P, Ehsan I, SME S, Nuijen B, TNM S, van Beurden M, Jordanova ES, JBAG H, van der Burg SH, Kenter GG. HPV16 E7 DNA tattooing: safety, immunogenicity, and clinical response in patients with HPV-positive vulvar intraepithelial neoplasia. Cancer Immunol Immunother. 2017:66:1163-73.

26. Miltz A, Price H, Shahmanesh M, Copas A, Gilson R. Systematic Review and Meta-Analysis of L1-VLP-Based Human Papillomavirus Vaccine Efficacy against Anogenital Pre-Cancer in Women with Evidence of Prior HPV Exposure. PLOS ONE. 2014;9(3):e90348. 